\title{
Slow evolution of elliptical galaxies induced by dynamical friction
}

\section{Non-adiabatic effects}

\author{
S. E. Arena ${ }^{1}$, G. Bertin ${ }^{1}$, T. Liseikina ${ }^{2}$, and F. Pegoraro ${ }^{3}$ \\ 1 Università degli Studi di Milano, Dipartimento di Fisica, via Celoria 16, 20133 Milano, Italy \\ e-mail: [Serena.Arena;Giuseppe.Bertin]@unimi.it \\ 2 Institute of Computational Technologies, SD RAS, Novosibirsk, Russia \\ e-mail: tatianavl@ngs.ru \\ 3 Università degli Studi di Pisa, Dipartimento di Fisica, largo Pontecorvo 3, 56100 Pisa, Italy \\ e-mail: pegoraro@df.unipi.it
}

Received 21 January 2006 / Accepted 14 March 2006

ABSTRACT

\begin{abstract}
Context. Many astrophysical problems, ranging from structure formation in cosmology to dynamics of elliptical galaxies, refer to slow processes of evolution of essentially collisionless self-gravitating systems. In order to determine the relevant quasi-equilibrium configuration at time $t$ from given initial conditions, it is often argued that such slow evolution may be approximated in terms of adiabatic evolution, for the calculation of which efficient semi-analytical techniques are available.

Aims. Here we focus on the slow process of evolution, induced by dynamical friction of a host stellar system on a minority component of "satellites", that we have investigated in a previous paper, to determine to what extent an adiabatic description might be applied. Methods. The study is realized by comparing directly $N$-body simulations of the stellar system evolution (in two significantly different models) from initial to final conditions in a controlled numerical environment.

Results. We demonstrate that for the examined process the adiabatic description is going to provide incorrect answers, not only quantitatively, but also qualitatively. The two classes of models considered exhibit generally similar trends in evolution, with one exception noted in relation to the evolution of the total density profile.

Conclusions. This simple conclusion should be taken as a warning against the indiscriminate use of adiabatic growth prescriptions in studies of structure of galaxies.
\end{abstract}

Key words. methods: $N$-body simulations - galaxies: elliptical and lenticular, $\mathrm{cD}$ - galaxies: evolution galaxies: kinematics and dynamics

\section{Introduction}

For the study of essentially collisionless systems the paradigm of adiabatic growth has received significant attention in astrophysics, especially in relation to two important contexts. Within cosmological scenarios of structure formation, it has been proposed as a natural framework to calculate the effects of infall of baryonic matter into the potential wells dominated by dark matter halos (see Blumenthal et al. 1986; Mo et al. 1988; Kochanek \& White 2001; Gnedin et al. 2004). In addition, it has been used as a tool to model stellar dynamical cusps in the vicinity of massive black holes at the center of galaxies (Young 1980; Goodman \& Binney 1984; Cipollina \& Bertin 1994; Quinlan et al. 1995; Merritt \& Quinlan 1998; van der Marel 1999). Either by semianalytical techniques or by means of suitable simulations, it has thus been shown that such adiabatic growth (usually justified by recalling that dissipative matter is able to accrete slowly towards the center) leads to a steepening of the underlying potential well.

Recently, in contrast with the above picture, several investigations have noted that processes usually interpreted in terms of dynamical friction, such as the capture of satellites and merging, tend to lead to systems with softer density profiles (El-Zant et al. 2001; Bertin et al. 2003, hereafter Paper I; El-Zant et al. 2004; Ma \& Boylan-Kolchin 2004; Nipoti et al. 2004), with a significant impact on the current debate about the observational counterparts to the universal halo density profiles found in cosmological simulations (Navarro et al. 1996; Moore et al. 1998; Ghigna et al. 2000; Navarro et al. 2004).

In Paper I we set up a numerical laboratory for the study of dynamical friction by simulating the slow evolution of a stellar system as a result of the interaction with a system of satellites, with a distribution that guarantees that approximate spherical symmetry is maintained. Such quasi-spherical model (inspired by the observation of globular cluster systems in some elliptical galaxies, but not necessarily meant to reproduce those conditions) has the advantage of being associated with a very smooth evolution, as desired if we wish to identify the small effects that are characteristic of dynamical friction; in this sense, it extends and improves earlier models, based on the capture of a single satellite, used to study the mechanism of dynamical friction (see Bontekoe \& van Albada 1987; Zaritsky \& White 1988; Bontekoe 1988; and many following papers).

The process of dynamical friction is not expected to follow the adiabatic picture, because the "microscopic" mechanisms at its basis (scattering of orbits in a discrete picture or waveparticle resonance in a continuum Vlasov description) are inherently non-adiabatic. In this paper we clarify this point by analyzing a set of controlled numerical experiments aimed at pointing out the differences between slow non-adiabatic and slow adiabatic evolution. For the general concepts and definitions relevant to the description of adiabatic processes in classical mechanics, 
the reader is referred to classical books such as Landau \& Lifchitz (1969, see Chap. 49), Goldstein (1980) (see Chap. 117), or advanced monographs such as Northrop (1963).

In Sect. 2 we describe the planned experiments and diagnostics, both at macroscopic and at microscopic level. In Sect. 3 we present the models and the code adopted in the numerical simulations. In Sect. 4 we describe the results, demonstrate the significance of non-adiabatic effects in phase space, and show that the evolution of the underlying distribution function of a stellar system can be qualitatively different in non-adiabatic models from that found in the adiabatic case. In Sect. 5 we draw the main conclusions. In the appendix we provide a theoretical framework to the adopted diagnostics of "scatter plots" by introducing a suitable description of the evolution of the relevant distribution function in phase space.

\section{Two experiments of slow evolution within quasi-spherical symmetry}

No dynamical evolution is expected for a stellar system in a (stable) collisionless equilibrium. But in the presence of some collisionality such steady state can be broken. This is the case for a stellar system that drags in, by dynamical friction, a heavy object or a system of heavy objects toward its center; while these "satellites" are dragged inwards, the reaction to the effects of dynamical friction makes the stellar system evolve (see Paper I), although very slowly. The question that we wish to clarify by means of a set of dedicated experiments is to what extent such slow evolution differs from a process in which a secular shift of matter toward the center of the stellar system occurs adiabatically.

To study this problem, following Paper I we refer to a spherically symmetric stellar system hosting a shell of total mass $M_{\text {shell }}$ fragmented into $N_{\text {shell }}$ heavy objects. In the continuum limit, of $N_{\text {shell }} \rightarrow \infty$ at fixed shell mass, the system would be characterized by spherical symmetry; for finite values of $N_{\text {shell }}$, the system is only approximately spherically symmetric. The symmetry of the system and the focus on a shell are chosen so as to better identify the small effects of slow evolution in the mechanisms that we wish to investigate.

In the following subsections we describe two experiments designed to study the differences between adiabatic and nonadiabatic processes.

\subsection{A shell distribution of satellites dragged in by dynamical friction (a non-adiabatic process)}

The first experiment, identified as case $A$, is the same as for runs $B S_{1}$ and $B S_{2}$ described in Paper I, that is a case when the evolution of the host galaxy proceeds as a result of the interaction with a discrete set of heavy objects falling in because of dynamical friction; while such a shell of satellites falls slowly toward the center, the galaxy rearranges its stellar orbits and therefore changes its distribution function.

The galaxy is taken to be described by a suitable equilibrium distribution function $f$. An initially smooth, spherically symmetric shell density distribution of matter, associated with the potential $\Phi_{\text {shell }}(r)$, is then added to the galaxy. We recall that, as described in Paper I (Sect. 3.2.2), the initial self-consistent galaxy potential $\Phi_{\mathrm{G}}(r)$, in the presence of the additional potential $\Phi_{\text {shell }}(r)$, is different from that associated with $f$ alone (i.e., in the absence of the shell), and has to be calculated separately to ensure that the initial state is indeed very close to dynamical equilibrium. The shell is then fragmented into $N_{\text {shell }}$ fragments (called also satellites) with initial velocities that are appropriate for test particles on circular orbits in the combined potential generated by the galaxy and the initially smooth shell $\left(\Phi_{\mathrm{G}}(r)+\Phi_{\text {shell }}(r)\right)$.

In the experiment, the satellites interact directly with the simulation particles that represent the collisionless host galaxy, and thus experience dynamical friction, spiraling in toward the center on a time-scale $\tau_{\text {fr }}$ much longer than the dynamical time $t_{\mathrm{d}}$. The evolution of the whole system is expected to be slow, but non-adiabatic.

\subsection{A similar shell distribution of matter moved in adiabatically}

The second experiment, identified as case $B$, considers the evolution of the same collisionless host galaxy considered in case $A$, but in the presence of an external collisionless shell of matter, which is moved (arbitrarily) toward the center of the galaxy mimicking the slow sinking of the fragmented shell of case $A$ with the same time-scale $\tau_{\mathrm{fr}} \gg t_{\mathrm{d}}$. In this case the dynamical friction process is not in action and the galaxy evolves because the external field experienced is (slowly) time-dependent.

In contrast with case $A$, here the shell is always kept smooth, spherically symmetric, not fragmented, and made to act on the galaxy uniquely as a slowly varying "external field". The evolution of the galaxy is thus expected to be, by construction, slow and adiabatic.

\subsection{Control cases}

We will also perform two additional experiments devised to test the dynamical evolution of the system when the mechanisms described above are not applicable.

The first control experiment, identified as case $C$, is a simulation that follows the prescription of case $B$, but with a much shorter time-scale $\tau_{\text {fast }}$, so as to break, on purpose, the condition of adiabatic evolution. This experiment is meant to better identify those properties that are characteristic of adiabatic evolution with respect to those associated with the driving by a general external forcing.

The second control experiment, case $D$, is a simulation to test the quality of the initial equilibrium in each of the above three cases $A, B$, and $C$. We consider two possibilities. One, in which the shell is treated as smooth, spherically symmetric, and collisionless, is better suited as a control test for case $A$; the shell is here represented by a large number of simulation particles placed on circular orbits but otherwise indistinguishable from the simulation particles used to represent the host galaxy. The other, in which the shell only acts as an "external field", is the more natural control test for cases $B$ and $C$. Case $D$, in addition to testing that the adopted initial conditions for the model of the galaxy and of the shell are correct, checks the accuracy of our simulations over long time-scales.

\subsection{Diagnostics}

To test the similarities and the differences in these cases, we resort to the following diagnostics. To study the problem at the macroscopic level, we show for the various cases the evolution of the galaxy density profile and of the global pressure anisotropy, defined as $2 K_{\mathrm{r}} / K_{\mathrm{T}}$, where $K_{\mathrm{r}}$ and $K_{\mathrm{T}}$ are the total kinetic energy of the galaxy in the radial and tangential direction respectively. 
Table 1. Expected conservation of single-particle quantities for the four cases considered (C: quantity conserved, NC: quantity not conserved).

\begin{tabular}{ccccc}
\hline \hline Case & $\mathrm{A}$ & $\mathrm{B}$ & $\mathrm{C}$ & $\mathrm{D}$ \\
\hline$E$ & $\mathrm{NC}$ & $\mathrm{NC}$ & $\mathrm{NC}$ & $\mathrm{C}$ \\
$J$ & $\mathrm{NC}$ & $\mathrm{C}$ & $\mathrm{C}$ & $\mathrm{C}$ \\
$I$ & $\mathrm{NC}$ & $\mathrm{C}$ & $\mathrm{NC}$ & $\mathrm{C}$ \\
\hline
\end{tabular}

At the microscopic level, to study the detailed conservations in phase-space, we produce "scatter plots" (final vs. initial quantities) for single-particle energy $E$, radial action $I$, and angular momentum $J$.

Ideally, in simulations belonging to case $A$, the three quantities, $E, I$, and $J$, are not conserved; in case $B$, the single-particle energy $E$ is not conserved, but $I$ and $J$ are; in case $C$, only $J$ is conserved. In case $D$, all quantities, $E, I$, and $J$, are conserved, and the relevant scatter plots thus define the limits of the numerical simulations (see Table 1).

The use of scatter plots raises interesting and important theoretical issues. Consider the scatter plot for the single-particle energy, which we already presented in the bottom right frame of Fig. 8 in Paper I and we will give in the upper frames of Figs. 3 and 4 of the present paper. This plot correlates the value of the energy of a single simulation particle at an initial time $t_{\text {in }}$ with that of the same simulation particle at a final time $t_{\text {fin. }}$. In an ideal code the simulation particles would follow the characteristics dictated by the collisionless Boltzmann (Vlasov) equation. Under conditions for which the energy is conserved, any initial distribution function would originate a distribution of simulation particles for which the scatter plot is a segment, for any value of $t_{\text {fin }}$; for a finite number of particles, the points would line up on such segment, covering the range from the minimum to the maximum value of particle energy, consistent with the adopted distribution function. Under conditions for which energy is not conserved, we may expect a distortion of that segment and, in addition, a broadening of the segment into a distribution of points, corresponding to a spread of effects, at given initial energy, associated with the different values of the angular momentum. Some conditions that determine the energy non-conservation may even determine a change in the value of the energy that depends on phase variables that define the orbit of the simulation particles, at given initial values of energy and angular momentum.

In real simulations, all the effects mentioned above are mixed with numerical effects, which we may generically call numerical noise. In other words, undesired numerical noise may be responsible for distortions of the segment-type plot and, more naturally, a general broadening with respect to the ideal case.

These considerations prompted us to move beyond the intuitive stage at which scatter plots are naturally appreciated, to see whether the information contained there can be set in a more satisfactory quantitative theoretical framework. An outline of such theoretical framework is provided in the appendix.

\section{The models and the code}

\subsection{The basic equilibrium of the host galaxy}

The basic model for the initial conditions of the host galaxy is constructed from two different distribution functions. The first is an $n=3$ polytrope with

$f(\boldsymbol{r}, \boldsymbol{v})=A\left(E_{0}-E\right)^{n-3 / 2}$

for $E<E_{0}$ and zero otherwise. The single particle total energy is $E=v^{2} / 2+\Phi(r)$ and $E_{0}=\Phi(R)=-G\left(M_{\mathrm{G}}+M_{\text {shell }}\right) / R$, where $M_{\mathrm{G}}$ and $R$ are respectively the mass and the radius of the galaxy and $M_{\text {shell }}$ is the mass of the shell. This is the galaxy model used in Paper I; it is convenient and is best suited for comparison with past investigations of the process of dynamical friction (for example, it was used by Bontekoe \& van Albada 1987, in their pioneering work on the subject).

The second is the $f^{(v)}$ distribution function

$f^{(v)}=A \mathrm{e}^{-\left[a E+d\left(J^{2} /|E|^{3 / 2}\right)^{v / 2}\right]}$

for $E<0$ and zero otherwise. Here $E$ and $J$ are the single-star energy and angular momentum. The four constants $A, a, d$, and $v$ are real and positive: from these one can identify two dimensional scales and two dimensionless parameters, such as $v$ and the dimensionless central potential $\Psi=-a \Phi(0)$. In this paper we will focus on the case $(v, \Psi)=(3 / 4,5)$ (see Trenti \& Bertin 2005 , for details about this distribution function and a discussion of the properties of the resulting self-consistent models for different values of $v$ and of $\Psi$ ). The resulting models are more concentrated than the $n=3$ polytrope and are characterized by an anisotropic pressure tensor. In general, these models provide a more realistic description of bright elliptical galaxies.

\subsection{The modified self-consistent equilibrium in the presence of a shell}

The initial shell is treated as smooth, spherically symmetric, and static, characterized by the density distribution:

$\rho_{\text {shell }}=\rho_{0} \exp \left[-4\left(r-r_{\text {shell }}(0)\right)^{2} / R_{\text {shell }}^{2}\right]$

for $\left|r-r_{\text {shell }}(0)\right| \leq R_{\text {shell }}$ (and vanishing otherwise); $r_{\text {shell }}(0)$ is the initial radial position of the shell, where its density is $\rho_{0}$, and $R_{\text {shell }}$ is its half-thickness.

As described in Sect. 3.2.2. of Paper I, in the presence of an external potential the distribution function of the galaxy differs, from that in absence of it, in the potential term that now is the total potential of the system: $\Phi(r)=\Phi_{\mathrm{G}}(r)+\Phi_{\text {shell }}(r)$. As a consequence, it is necessary to re-compute the equilibrium models of the galaxy by solving the Poisson equation for the self-consistent $\Phi_{\mathrm{G}}(r)$ in the presence of the external shell density distribution.

\subsection{The code}

The galaxy evolution is described by a collisionless (mean-field) particle-mesh code, which is an improved version of the original van Albada (1982) code. The new code (see Trenti et al. 2005; and Trenti 2005) is based on an improved Poisson solver scheme, in which the angular grid is dropped, while the radial grid is adaptive so that each cell is populated by approximately the same number of particles.

The galaxy is sampled with $N$ simulation particles, with initial positions and velocities extracted from the distribution functions described in Sect. 3.2 by means of Monte Carlo methods (see Paper I). The same method is applied to derive the initial positions of the $N_{\mathrm{f}}$ fragments of the shell, of mass $M_{\mathrm{f}}=$ $M_{\text {shell }} / N_{\mathrm{f}}$. These fragments or satellites are described by an extended Plummer density profile:

$\Phi_{\mathrm{f}}(r)=-\frac{G M_{\mathrm{f}}}{\left(R_{\mathrm{f}}^{2}+r^{2}\right)^{1 / 2}}$,

where $R_{\mathrm{f}}$ is the radius of each shell fragment. In the simulations of case $A$ (see Sect. 2.1), the equations that describe the interactions among galaxy simulation particles and shell fragments are 
those described in Paper I. Each particle of the galaxy feels the action of the whole galaxy via its mean field and that of each shell fragment directly; each shell fragment feels the sum of the direct actions from all the galaxy simulation particles and of the direct actions from the other shell fragments.

In the simulations of cases $B$ and $C$, there are no discrete objects (satellites) involved. Therefore, the simulation particles follow the orbits dictated by the mean field generated by the galaxy, as in case A, together with the force of the shell which is treated as an external, spherically symmetric, and smooth force.

For the diagnostics mentioned in Sect. 2.4, the radial action $I$ of a single simulation particle, at time $t$, is computed in a semi-analytical way, starting from the values of $E$ and $J$. The total potential $\Phi(r)=\Phi_{\mathrm{G}}(r)+\Phi_{\text {shell }}(r)$, felt by the particle, is first measured in the simulation at time $t$. Then, the effective potential $\Phi_{\text {eff }}(r ; J)=\Phi(r)+J^{2} /\left(2 r^{2}\right)$, the radial velocity $v_{\mathrm{r}}(r ; E, J)=\sqrt{2\left[E-\Phi_{\mathrm{eff}}(r)\right]}$, and the two turning points $r_{\min }(E, J)$ and $r_{\max }(E, J)$ are derived. Finally, the radial action is found by numerical integration: $I(E, J)=$ $2 \int_{r_{\min }}^{r_{\max }} v_{\mathrm{r}}(r ; E, J) \mathrm{d} r$.

\subsection{Units}

The units adopted are $10 \mathrm{kpc}$ for length, $10^{11} M_{\odot}$ for mass, and $10^{8} \mathrm{yr}$ for time. Thus, velocities are measured in units of $97.8 \mathrm{~km} \mathrm{~s}^{-1}$ and the value of the gravitational constant $G$ is $\approx 4.497$.

\section{Numerical simulations}

The galaxy is sampled with $N=250000$ simulation particles and has a mass $M_{\mathrm{G}}=2$ and a half-mass radius $r_{\mathrm{M}}=0.3$ (in code units); simulations with higher values of $N$ have also been run. We should recall that the issues of the reliability (stability) of the code and of the adequacy of the number of particles used in the simulations were already addressed in previous papers. In particular, in Paper I (see Sect. 5.1 of that paper) we ran a simulation of a single sinking satellite with $N$ up to $2 \times 10^{6}$ and found very little change in the observed evolution, down to the innermost radii reached by the infalling satellite; in addition (see Sect. 4.4 .2 of that paper), we checked, with general qualitative agreement, the main results obtained earlier by Bontekoe \& van Albada (1987), who had originally investigated the process of dynamical friction with only a few thousand simulation particles. Furthermore, the new version of the code used in this paper has been extensively tested with many simulations with $N \geq 8 \times 10^{5}$, especially designed to check its stability in reproducing concentrated models over long time scales; the new version of the code has also been tested against the performance of high-quality tree codes currently available (Trenti 2005; Trenti et al. 2005). To be sure, if the process of dynamical friction depended too sensitively on detailed resonant effects, even with these large numbers of simulation particles the phase space might be insufficiently well sampled.

The mass of the shell is one tenth of that of the galaxy $\left(M_{\text {shell }}=0.2\right)$. The shell is initially located at $r_{\text {shell }}(0)=r_{\mathrm{M}}$. The radius of each fragment is equal to the half thickness of the shell, $R_{\mathrm{f}}=R_{\text {shell }}=0.1$, that is one third of the half-mass radius of the galaxy. These values of $M_{\text {shell }} / M_{\mathrm{G}}$ and of $R_{\mathrm{f}} / r_{\mathrm{M}}$ repeat the conditions of previous investigations (Bontekoe \& van Albada 1987; Paper I); this choice helps to better bring out the effects of dynamical friction within the computational resources used.
Table 2. The main set of numerical simulations; $n=3$ for the polytropic model and $(v, \Psi)=(3 / 4,5)$ for the $f^{(v)}$ model (see text for description); in runs $D 1$ and $D 3, N_{\mathrm{f}}$ represents the number of simulation particles treated as collisionless.

\begin{tabular}{ccccc}
\hline \hline Case & Process & Run & $\begin{array}{c}\text { Shell } \\
\text { type }\end{array}$ & $\begin{array}{c}\text { Galaxy } \\
\text { model }\end{array}$ \\
\hline A & Dynamical & A1 & $N_{\mathrm{f}}=20$ & Polytrope \\
& friction & A2 & $N_{\mathrm{f}}=100$ & Polytrope \\
& & A3 & $N_{\mathrm{f}}=20$ & $f^{(v)}$ \\
& & A4 & $N_{\mathrm{f}}=100$ & $f^{(v)}$ \\
B & External & B1 & External slow & Polytrope \\
& adiabatic & B2 & External slow & $f^{(v)}$ \\
C & External & C1 & External fast & Polytrope \\
& non & C2 & External fast & $f^{(v)}$ \\
& adiabatic & & & \\
D & Control & D1 & $N_{\mathrm{f}}=25000$ & Polytrope \\
& & D2 & External static & Polytrope \\
& & D3 & $N_{\mathrm{f}}=25000$ & $f^{(v)}$ \\
& & D4 & External static & $f^{(v)}$ \\
\hline
\end{tabular}

With the above choice of $r_{\mathrm{M}}$ and $M_{\mathrm{G}}$, the dynamical time of the galaxy without the shell, defined as $t_{\mathrm{d}}=G M_{\mathrm{G}}^{5 / 2} /\left(-2 E_{\mathrm{tot}}\right)^{3 / 2}$, is $t_{\mathrm{d}}^{(\mathrm{pol})}=0.1476$ for the polytropic model and $t_{\mathrm{d}}^{(v)}=0.1881$ for the $f^{(v)}$ model (in code units). To better illustrate the meaning of $t_{\mathrm{d}}$, we note that the period of two selected circular orbits, at radii $1.17 r_{\mathrm{M}}$ and $3.3 r_{\mathrm{M}}$, is $5.4 t_{\mathrm{d}}$ and $14.3 t_{\mathrm{d}}$ for the polytropic model and $3.0 t_{\mathrm{d}}$ and $12.7 t_{\mathrm{d}}$ for the $f^{(v)}$ model.

In all simulations the conservation of the total energy and total angular momentum is respectively of the order of $10^{-6}$ and $10^{-5}$ per dynamical time, except for case $D$, for the polytropic model, in which the conservation is better by one order of magnitude.

The evolution of the system is followed up to a final time, $t_{\text {fin }}=90 t_{\mathrm{d}}$ (for the fast case $C$, the evolution is followed up to $\left.t_{\text {fin }}=2 t_{\mathrm{d}}\right)$. The configuration of the system at such final time is compared with that at $t_{\text {in }}=0.14 t_{\mathrm{d}}$.

The list of experiments is given in Table 2 .

\subsection{Case A: fall of a shell of satellites by dynamical friction}

We have performed four simulations of this type: two with the host galaxy modeled by a polytropic distribution function and the shell sampled with 20 and 100 fragments (runs $A 1$ and $A 2$, respectively) and two with the galaxy described by the $f^{(v)}$ model and the same configurations for the shell (runs $A 3$ and $A 4$ ). Runs $A 1$ and $A 2$ basically repeat runs $B S_{1}$ and $B S_{2}$ described in Paper I, but with the new improved code (Paper I made use of the original van Albada (1982) code).

In these runs, because of the dynamical friction experienced by the individual fragments, the shell of fragments sinks in slowly, toward the center of the galaxy, while becoming thicker in radius, exchanging part of its energy with the galaxy (see top panel of Fig. 1 for run A1). For the same galaxy model, by reducing the number of fragments (from 100 to 20) the fall of the shell is faster, as expected because the fragments have larger masses, and the thickening of the shell is less pronounced.

The host galaxy responds with an expansion that leads to a density profile broader in the outer parts and shallower in the inner regions. The degree of variation of the density profile from the initial to the final configuration increases when the number of shell fragments is decreased (from about $11 \%$ of run $A 1$ to about $2 \%$ of run $A 2$, for the polytropic model) and the size of 

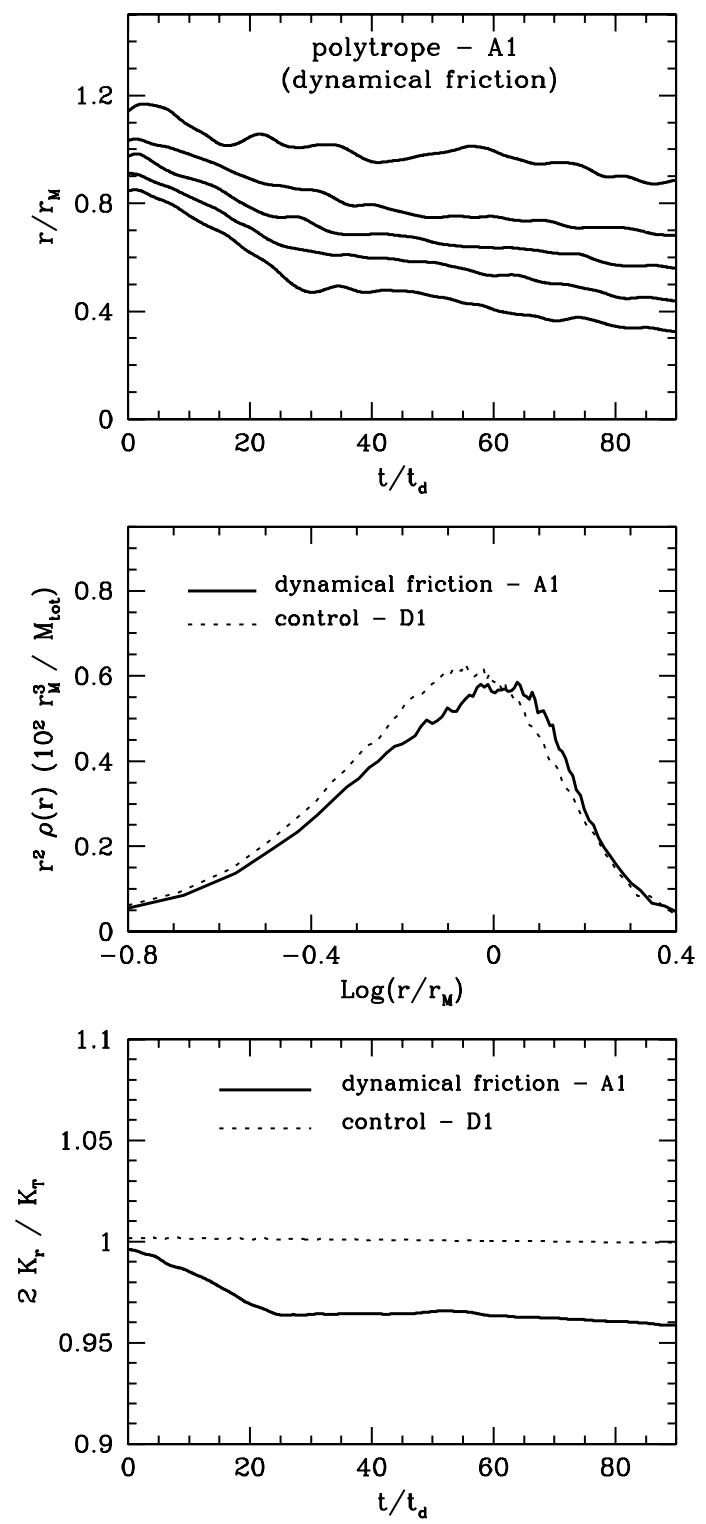

Fig. 1. Case A (dynamical friction): collisional shell of $N_{\mathrm{f}}$ fragments in an $n=3$ polytropic galaxy (run $A 1$ ). The top panel describes the time evolution of the radii of the spheres containing $15,35,55,75,95 \%$ of the total shell mass. The central panel compares the final galaxy density profile to that of the corresponding control case, which is very close to the initial galaxy density profile. The bottom panel illustrates the time evolution of the galaxy global anisotropy parameter $\left(2 K_{\mathrm{r}} / K_{\mathrm{T}}\right)$.

the effects changes with the galaxy model (from about $11 \%$ of run $A 1$ to about $25 \%$ of run $A 3$ for $N_{\mathrm{f}}=20$ ). The initial and final density profiles observed in run $A 1$ are illustrated in the central panel of Fig. 1; the plot makes use of the volume-weighted density $r^{2} \rho(r)$ so as to better bring out the shift of mass associated with the observed evolution.

In addition, the host galaxy responds by changing its pressure tensor in the tangential direction; the effect is more marked in cases $A 1$ and $A 3$, which have a smaller number of fragments. For the polytropic model, the final values of the global anisotropy parameter $2 K_{\mathrm{r}} / K_{\mathrm{T}}$ (twice the ratio of the total kinetic energy in the radial direction to that in the tangential directions) are 0.96 and 0.98 respectively for runs $A 1$ and $A 2$ (see bottom panel of Fig. 1 for run $A 1$ ), while the initial configuration starts as isotropic (with $2 K_{\mathrm{r}} / K_{\mathrm{T}}=1$ ). In turn, the $f^{(v)}$ model, which is
Table 3. Conservation of single-particle quantities. For the various runs of cases $A$ and $B$ the table lists the percentage of galaxy particles with $\left|\left(Q_{\text {fin }}-Q_{\text {in }}\right) / Q_{\text {in }}\right|<0.08$ for the quantities $Q=E, J$, and $I$. For case $C$ the "conservation level" is lowered to $5 \%$, because the scatter is smaller due to the short duration of the simulations. For each case $(A, B$, and $C)$ the numbers are listed close to those of the corresponding control case $D$ (cf. the expectations of Table 1). The worse conservation of $J$ and $I$ with respect to that of $E$ depends on the fact that a significant number of particles have initial values of $J$ and $I$ near zero.

\begin{tabular}{|c|c|c|c|c|c|c|c|}
\hline $\begin{array}{c}\text { Case } \\
t_{\text {in }}-t_{\text {fin }}\end{array}$ & $\begin{array}{r}\mathrm{D} \\
0 \\
\end{array}$ & $14-90$ & & $\begin{array}{c}\mathrm{D} \\
0.1 \\
\end{array}$ & $\begin{array}{c}\mathrm{B} \\
90 t_{\mathrm{d}}\end{array}$ & $\begin{array}{c}\mathrm{D} \\
0.1 \\
\end{array}$ & $\begin{array}{r}\mathrm{C} \\
2 t_{\mathrm{d}} \\
\end{array}$ \\
\hline \multicolumn{8}{|c|}{ Polytrope $(n=3)$} \\
\hline Runs & D1 & A1 & A2 & $\overline{\mathrm{D} 2}$ & B1 & $\mathrm{D} 2$ & $\overline{\mathrm{C} 1}$ \\
\hline$E$ & 93 & 38 & 62 & 98 & 58 & 100 & 28 \\
\hline$J$ & 61 & 23 & 34 & 68 & 67 & 98 & 98 \\
\hline$I$ & 38 & 15 & 24 & 42 & 41 & 83 & 55 \\
\hline \multicolumn{8}{|c|}{$f^{(v)}(v, \Psi)=(3 / 4,5)$} \\
\hline Runs & D3 & A3 & $\mathrm{A} 4$ & $\mathrm{D} 4$ & $\mathrm{~B} 2$ & D4 & $\overline{\mathrm{C} 2}$ \\
\hline$E$ & 89 & 30 & 57 & 91 & 42 & 100 & 29 \\
\hline$J$ & 55 & 20 & 32 & 60 & 60 & 89 & 89 \\
\hline$I$ & 46 & 18 & 31 & 48 & 48 & 83 & 54 \\
\hline
\end{tabular}

characterized by a radially biased pressure anisotropy to begin with, slowly evolves toward a more isotropic configuration.

Because collisions are at the basis of the underlying physical mechanism of dynamical friction, we expect to see their effects on the particles of the galaxy that have taken part in the exchange of energy and angular momentum with the fragments of the shell. For the polytropic model, by comparing the first column of Fig. 3 with the first column of Fig. 4, which represents the control case, one indeed sees that the single-particle energy $E$, angular momentum $J$, and radial action $I$ (of the galaxy simulation particles) are not conserved. The percentage of particles with quantities conserved to a given level increases with $N_{\mathrm{f}}$. In the polytropic galaxy, about $66 \%$ and $45 \%$ (respectively for $N_{\mathrm{f}}=100$ and 20) of the particles have $\left|\left(E_{\mathrm{fin}}-E_{\text {in }}\right) / E_{\text {in }}\right|<0.08$, where $E_{\text {in }}$ and $E_{\text {fin }}$ are the initial and final energy values, in contrast with the value of $94 \%$ observed in the collisionless control case $D$. The corresponding values for $J$ and $I$ and for the runs related to the $f^{(v)}$ model are shown in Table 3. (The generally worse conservation of $J$ and $I$ with respect to that of $E$ depends on our definition of conservation in terms of relative variations and on the fact that a significant number of particles have values of $J$ and $I$ near zero. For example, in the control run $D 1$, if we focus only on particles with $J / J_{\max }$ and $I / I_{\max }$ greater than 0.25 (where $J_{\max }$ and $I_{\max }$ are the maximum values of $J$ and $I$ taken by the galaxy particles), the percentage of conservation increases respectively from 61 to 76 and from 38 to 65 (the fraction of particles left out is $32 \%$ and $70 \%$ ).)

\subsection{Case B: fall of a shell described by a slow external potential}

We have performed two simulations of this type: run $B 1$ for the polytropic galaxy model and run $B 2$ for the $f^{(v)}$ model.

The construction of the external force mimicking the fall of a shell is based on the results for the shell density distribution with $N_{\mathrm{f}}=20$ observed in runs $A 1$ and $A 3$. The external specific radial force to which a galaxy particle, at radius $r$ and at time $t$, is subject is given by $f_{r}(r, t)=-G_{\text {shell }}(r, t) / r^{2}$, where $M_{\text {shell }}(r, t)=M_{\text {shell }}(r, t=0)\left(t_{\text {fin }}-t\right) / t_{\text {fin }}+M_{\text {shell }}\left(r, t=t_{\text {fin }}\right)\left(t / t_{\text {fin }}\right)$ is the mass of the shell inside the sphere of radius $r$ at time $t$. 

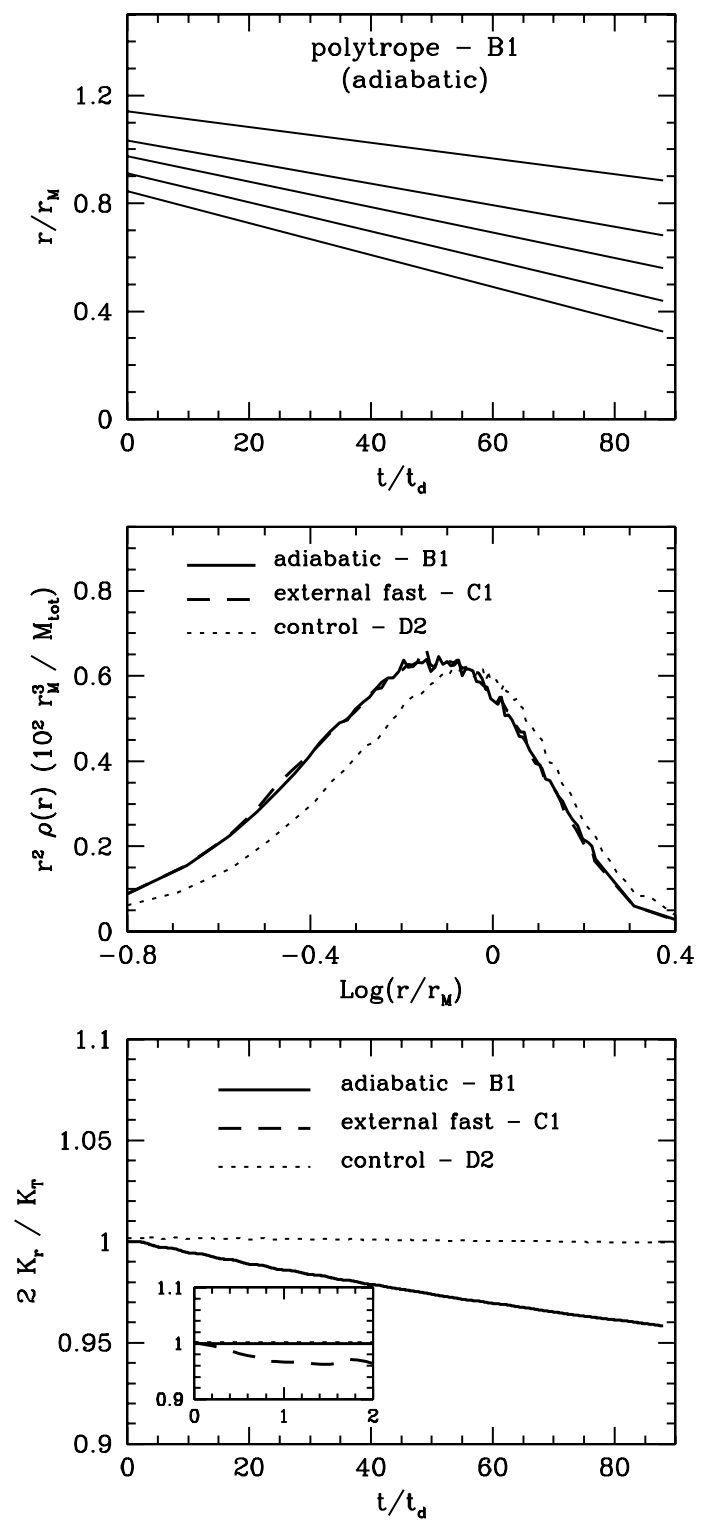

Fig. 2. Cases B (slow and adiabatic) and C (fast, non-adiabatic): evolution of an $n=3$ polytropic galaxy in the presence of an external potential which mimics the fall of the shell observed in run $A 1$. The three panels describe the same quantities as in Fig. 1. Note the qualitative difference with case $A$ in the evolution of the density profile. In the middle frame the $B$ and $C$ curves are basically identical. In the bottom frame the fast case $C 1$ is illustrated only in the insert.

The values of $M_{\text {shell }}(r, t=0)$ and $M_{\text {shell }}\left(r, t=t_{\text {fin }}\right)$ are computed in the runs $A 1$ and $A 3$ (for run $A 1$, see top panel of Fig. 2).

The observed evolution of the galaxy in the presence of the slow change of the potential associated with the external shell is very different from that in response to the direct interaction with the fragments of the shell. This is a purely collisionless case in which the galaxy energy changes slowly, much like in cases $A$, but in the absence of dynamical friction.

The density distribution becomes more peaked in the inner regions and shallower in the outer parts (see central panel of Fig. 2). For a given galaxy model, the effect is found to be stronger when a comparison is made with a case of dynamical friction characterized by the lower value of $N_{\mathrm{f}}\left(N_{\mathrm{f}}=20\right)$. At fixed value of $N_{\mathrm{f}}$, the effect is stronger for the $f^{(v)}$ model.
The total kinetic energies of the galaxy $K_{\mathrm{r}}$ and $K_{\mathrm{T}}$ (in the radial and in the tangential directions) increase with time, because of the energy change associated with the time-dependent process, but at different rates, so that the galaxy global pressure anisotropy evolves in the tangential direction. Much like in runs of case $A$, the final configuration of the polytrope is tangentially anisotropic (with a final global parameter $2 K_{\mathrm{r}} / K_{\mathrm{T}}=0.96$; see bottom panel of Fig. 2). A similar trend is found for the $f^{(v)}$ model.

At the microscopic level, as expected, the single-particle energy is not conserved because the potential in which the particles move is time-dependent. On the other hand, the angular momentum and the radial action are conserved, within the numerical limits (see second column of Figs. 3 and 4 and Table 3). A similar behaviour is found for the $f^{(v)}$ model.

\subsection{Case C: fall of a shell described by a fast external potential}

As for case $B$, we have performed two simulations of this type: run $C 1$ for the polytropic galaxy model and run $C 2$ for the $f^{(v)}$ model.

In these simulations the external shell is moved in, from the initial to the final state, as in case $B$, but at a much faster speed, that is in only two dynamical times. This rapid variation leads to a final configuration characterized by a density distribution (see central panel of Fig. 2) and global pressure anisotropy (see bottom panel of Fig. 2) similar to those observed in case $B$. In particular, the curves labelled $B 1$ and $C 1$ in the middle frame of Fig. 2 overlap, showing that the density response of the galaxy to the externally imposed field mimicking the infall of a shell does not depend on whether the process is actually slow or fast. A similar behaviour is observed in the study of the $f^{(v)}$ model.

At the microscopic level, for both galaxy models, the singleparticle energy and radial action are not conserved, while the angular momentum is because of the spherical symmetry. The numerical scatter observed in the relevant scatter plots is relatively small because of the short duration of the simulation (see third column of Figs. 3 and 4 and Table 3).

\subsection{Case D: control runs with a collisionless and with a static external shell}

We have performed four simulations of this type: runs $D 1$ and $D 2$ for the polytropic galaxy model and runs $D 3$ and $D 4$ for the $f^{(v)}$ model.

These simulations have been performed to test the stability of the initial conditions of the system considered in cases $A, B$, and $C$. In runs $D 1$ and $D 3$, designed to test runs $A 1-A 4$, the shell is sampled with 25000 particles, so that each shell simulation particle has the same mass as each galaxy simulation particle; the shell particles are then treated as collisionless, interacting with one another and with the the particles of the galaxy via the mean field. In runs $D 2$ and $D 4$, designed to test runs $B 1$, $B 2, C 1$, and $C 2$, the shell is treated as an external field, much like in cases $B$ and $C$, but kept static, in its initial configuration $M_{\text {shell }}(r, t=0)$.

The initial "equilibrium" configuration of the host galaxy in the presence of the collisionless or static shell is checked to stay basically unchanged in the course of the simulations. For both galaxy models, in run $D 1$ and $D 3$, the radii of the spheres containing $15,25,35,45,55,65,75,85,95 \%$ of the total mass of the shell remain constant in time to within $1 \%$, except for that 
POLYTROPIC MODEL $(\mathrm{n}=3)$
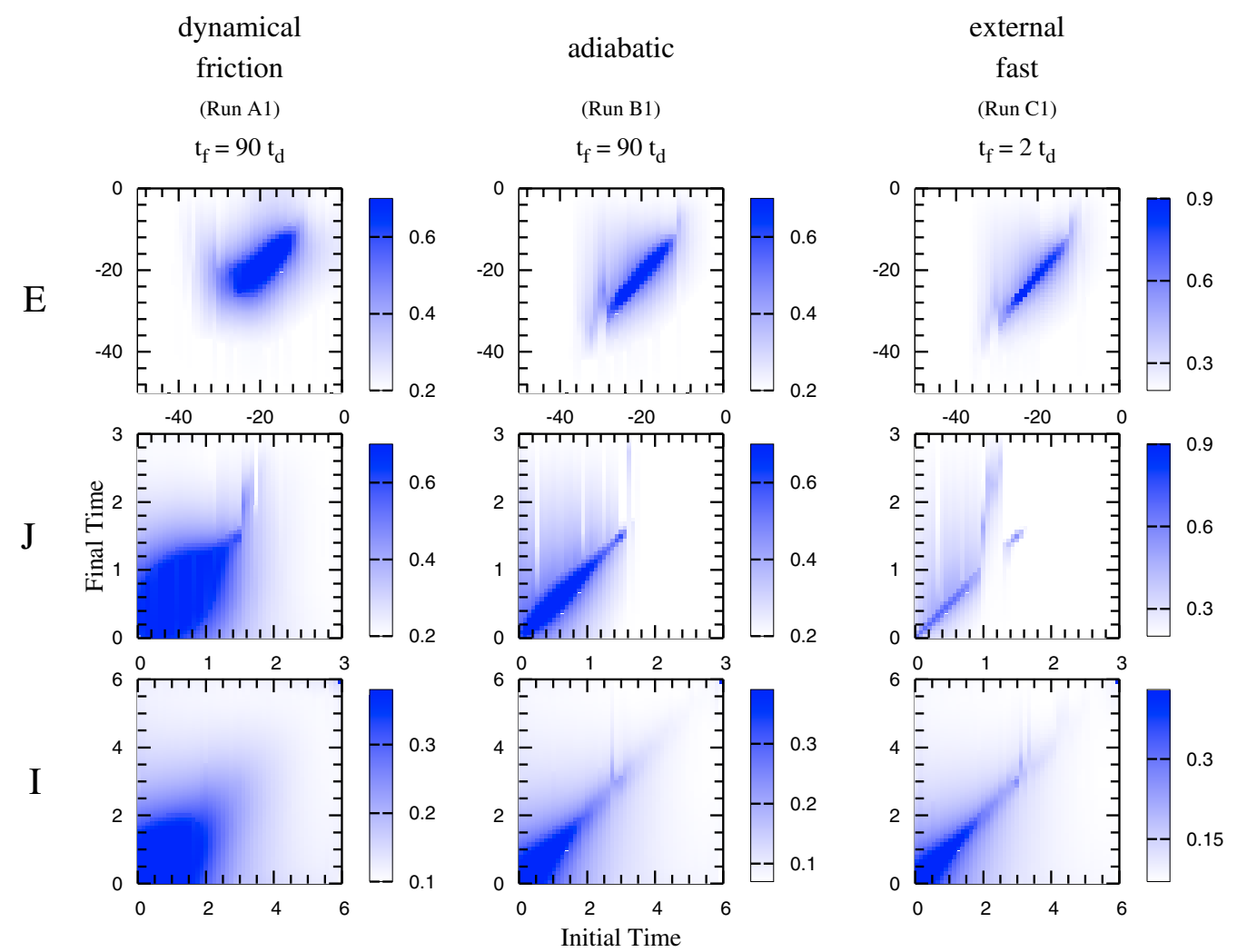

Fig. 3. Correlation between single-particle quantities at an initial time $\left(t_{\text {in }}=0.14 t_{\mathrm{d}}, x\right.$-axis) and at a final time ( $t_{\text {fin }}, y$-axis): energy (top row), angular momentum (central row), and radial action (bottom row), expressed in code units (see Sect. 3.4). The first column displays the results for case $A$ (run $A 1 ; t_{\text {fin }}=90 t_{\mathrm{d}}$ ), the second for case $B$ (run $B 1 ; t_{\text {fin }}=90 t_{\mathrm{d}}$ ), the third for case $C$ (run $C 1 ; t_{\text {fin }}=2 t_{\mathrm{d}}$ ); the corresponding control cases are displayed in Fig. 4. For each quantity $Q$, the grey scale used represents the number density of simulation particles in the plane $Q_{0}-Q$. By comparing each panel with the corresponding panel in Fig. 4, the conservation properties listed in Table 1 (and quantified in Table 3), are confirmed.

containing $5 \%$ that remains constant only to $4 \%$. A similar diagnostics for the galaxy simulation particles shows a conservation better than $0.5 \%$; in the innermost regions $\left(r<0.1 r_{\mathrm{M}}\right)$, because of the relatively small number of particles that are involved, the density profiles are found to be steady to within $2 \%$. The changes in the total kinetic energy of the galaxy in the radial and tangential directions are found to be less than $0.3 \%$.

In runs $D 2$ and $D 4$ the overall stability is checked to be even better than for runs $D 1$ and $D 3$ : the mass radii remain constant to within $0.05 \%$ (except for the $5 \%$ mass radius for which the stability is to $0.2 \%$ ), the density profile remains unchanged to within $1 \%$, and the total kinetic energies are stable to less than $0.1 \%$.

Because of these control cases are designed to be spherically symmetric and time-independent, the single particle quantities (energy $E$, angular momentum $J$, and radial action $I$ ) should be conserved. The level of conservation observed over the entire period of the simulation, thus illustrating the accuracy of our code, is shown in Fig. 4 and in Table 3.

For completeness, we have also run one additional control case, D5, of a pure polytropic model, with no shell, to test whether some of the scatter present in the control cases $D$ might be associated with the introduction of the shell itself. The relevant conservation levels over the time interval $0.14 t_{\mathrm{d}}-90 t_{\mathrm{d}}$, corresponding to the $D 2$ column at the center of Table 3 , are 97 (for $E$ ), 66 (for $J$ ), and 41 (for $I$ ), practically identical to the corresponding values for run $D 2$.

\subsection{One qualitative difference in behavior between the polytropic and the $f^{(v)}$ model}

We have noted that, as a result of the slow evolution associated with the process of dynamical friction (see middle frame of Fig. 1), the density distribution of the galaxy is softened, with the mass being basically transported outwards, in contrast with the trend associated with the corresponding adiabatic case (see middle frame of Fig. 2). This general property is found in both the polytropic and the $f^{(v)}$ models that we have investigated.

If we focus instead on the evolution of the total density profile (galaxy + shell), we note a curious qualitative difference in the behavior of the two models. In fact, while for the polytropic model (characterized initially by a broad core) the evolution induced by dynamical friction leads to a higher concentration in the total density profile, for the $f^{(v)}$ model (which starts with higher concentration) the softening and broadening of the density profile occurs not only for the galaxy density distribution but also for the total density profile (see Fig. 5). In all the cases studied, the adiabatic evolution is associated with the most concentrated final density profiles.

These considerations are especially relevant when one imagines astrophysical applications based on the study of rotation curves in the innermost regions of galaxies. We do not have a simple physical argument to explain the observed difference in behavior, which was noted as a side-result in this project dedicated to studying the non-adiabatic character of dynamical 


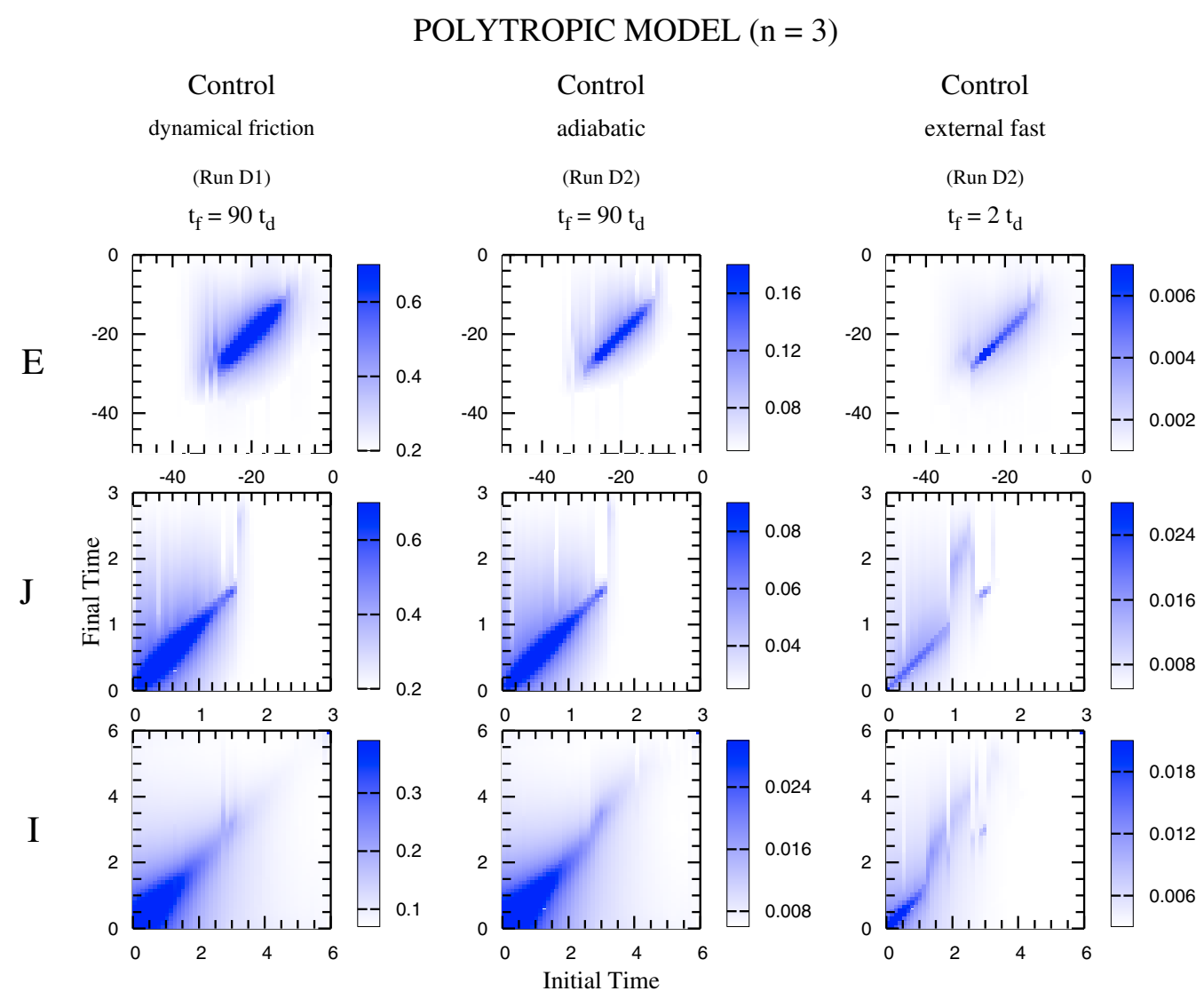

Fig. 4. Correlations observed in the control runs $D 1$ and $D 2$, defining the accuracy of the code. The layout and the notation are similar to those of Fig. 3. The apparent thinness of the scatter plots in the third column of this figure and in Fig. 3 are due to the shortness of the run presented $\left(t_{\text {fin }}=2 t_{\mathrm{d}}\right)$.

friction. We are planning to return to this issue in a separate paper that will be devoted to comparing the process of dynamical friction in models characterized by a broad core to the process occurring in concentrated models.

\section{Conclusions and perspectives}

In this paper we have studied in detail the slow evolution of quasi-collisionless self-gravitating systems by comparing the situation when such evolution is due to effects of dynamical friction to a situation that is strictly adiabatic but otherwise similar. The two types of evolution have been studied by specially designed numerical experiments and have been compared both at the macroscopic and at the microscopic level.

At the microscopic level we have checked that the relevant single-particle quantities, such as energy $E$, angular momentum $J$, and radial action $I$, are indeed conserved or not conserved as expected from first principles. These studies have been quantified by means of suitable "scatter plots" which bring out interesting structural properties of the collisionless Boltzmann (Vlasov) equation, when suitably expressed in terms of evolution variables (see Appendix).

At the macroscopic level, we find a qualitatively different behaviour in the evolution of the density distribution of the stellar system under investigation. In particular, in the (non-adiabatic) case of slow evolution resulting from dynamical friction, the density profile becomes "softer" in the course of evolution, in contrast with the overall steepening and contraction noted in the adiabatic case. In turn, the overall evolution of pressure anisotropy turns out to be similar in the two cases.

In this study we have considered the evolution induced on the host galaxy by a slow infall of matter distributed in a relatively thin shell. This paper is part of a longer-term project, currently in progress, where we aim at studying the more general problem in which the infalling matter has a full three-dimensional distribution. By comparing such more general behavior with the behavior that may be anticipated by superposing the effects of adjacent thin shells of matter (now described quantitatively in this paper), we will be able to assess to what extent the general problem of infall can be reduced to a local analysis or else whether full global simulations are generally required, with a behavior that may depend on a case by case basis. It would be important to identify general trends in such general process, even if those trends will be different from those suggested by the picture of adiabatic infall.

The astrophysical applications of this long-term project are basically directed on two goals. Specifically in the galactic context, we wish to develop a quantitative picture of the effects on a stellar system (such as a bulge or an elliptical galaxy) associated with the slow infall of a system of globular clusters; similar effects may operate in determining a more realistic picture of galaxy formation, in which the capture of a large number of satellites (minor mergers) may accompany or follow the process of incomplete violent relaxation (known to occur in purely collisionless systems). In the more general cosmological context, we wish to assess whether dynamical friction can change significantly the scenario of cusp formation, so prominently present 

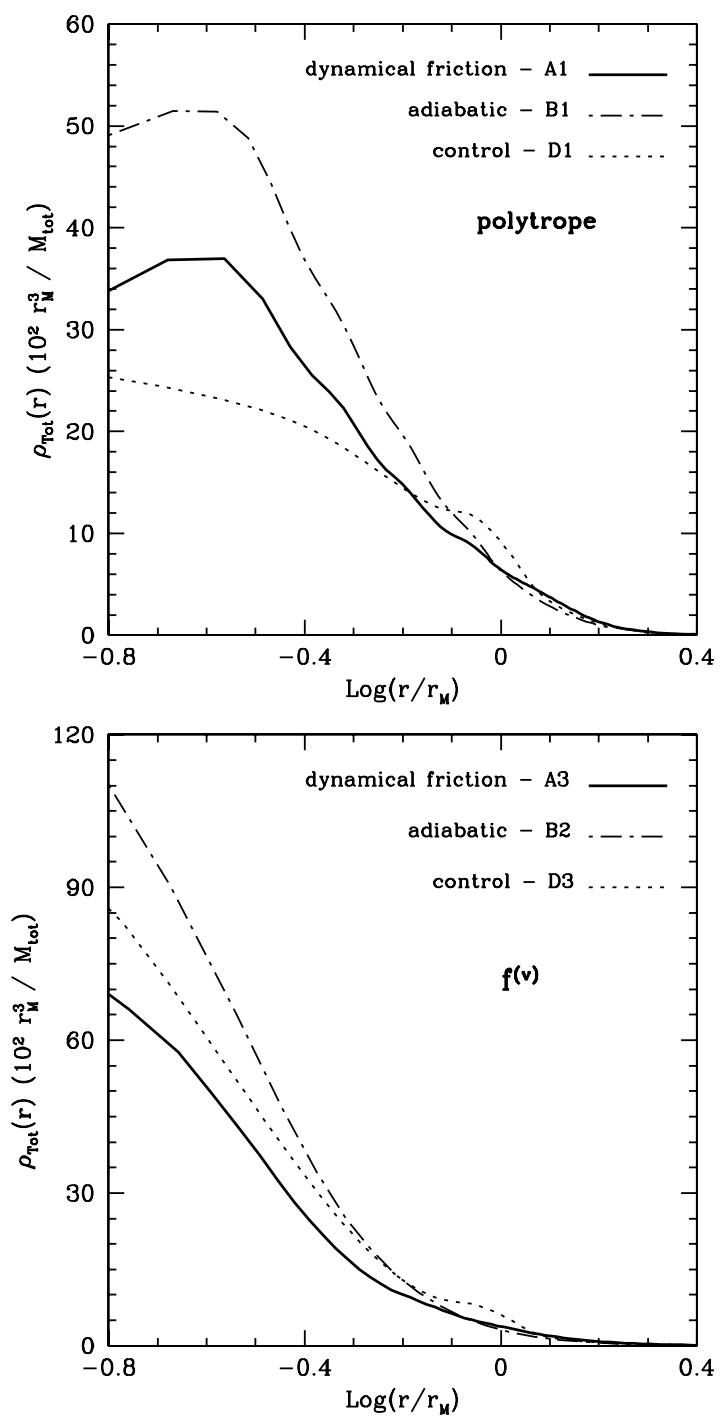

Fig. 5. Comparison of final total density profiles in different evolution processes (solid lines, dynamical friction; dotted lines, control cases; dash-dotted lines, adiabatic evolution) for the polytropic galaxy model (upper frame) and for the $f^{(v)}$ model (lower frame). The control cases, which are basically time-independent, thus illustrate the adopted initial conditions.

in cosmological simulations. For addressing properly all these issues we will have to extend the calculations so as to allow for the evaporation and possible tidal disruption of the satellites that are dragged in by dynamical friction.

Acknowledgements. We would like to thank Michele Trenti for providing us with his improved code for the simulations and Luca Ciotti, Michele Trenti, and Tjeerd van Albada for a number of useful suggestions. We are also glad to thank Alessandro Romeo for his constructive criticism. Part of this work was supported by the Italian MIUR.

\section{Appendix A}

We start by examining the origin of distortions and dispersion in scatter plots for the ideal case in which the simulation particles follow rigorously and exactly the characteristics of the collisionless Boltzmann equation. To set a quantitative framework for the corresponding effects associated with the numerical noise is far more difficult and beyond the scope of the present paper. The main idea that we follow below consists in reading the scatter plots as suitable cuts (or projections) of a distribution function expressed in terms of evolution variables.

A formal definition of the scatter plots mentioned above can be given in terms of the distribution function $f=f(\boldsymbol{x}, \boldsymbol{p}, t)$ of a stellar system (i.e., more precisely of its mass density distribution function). Here $\boldsymbol{x}, \boldsymbol{p}$ are the 6 position and momentum coordinates in phase space and $f(\boldsymbol{x}, \boldsymbol{p}, t) \mathrm{d} \boldsymbol{x} \mathrm{d} \boldsymbol{p}$ is the mass inside the phase volume $\mathrm{d} \boldsymbol{x} \mathrm{d} \boldsymbol{p}$. The distribution $f$ obeys the collisionless Boltzmann equation

$\frac{\partial f}{\partial t}+\{f, H\}_{x, p}=0$

where $H=H(x, p, t)$ is the single-star Hamiltonian that includes the effect of external forces such as those arising from the presence of the falling satellites and $\{,\}_{x, p}$ are the standard Poisson brackets (referred to the variables $\boldsymbol{x}$ and $\boldsymbol{p}$ ). As is well known, Eq. (A.1) can be rewritten in the Lagrangian form

$\frac{D f}{D t}=0$,

which expresses the fact that the distribution function remains constant as it evolves in time along the characteristics of the Hamiltonian $H$. In turn, the general solution of Eq. (A.2) is $f(\boldsymbol{x}, \boldsymbol{p}, t)=F\left(\boldsymbol{x}_{0}, \boldsymbol{p}_{0}\right)$ where $\boldsymbol{x}_{0}, \boldsymbol{p}_{0}$ are the phase-space coordinates at $t=0$ of the characteristics that at time $t$ reach $\boldsymbol{x}, \boldsymbol{p}$ and $F$ denotes the assigned initial condition for the (positivedefinite) distribution function at $t=0$, i.e. $f\left(\boldsymbol{x}_{0}, \boldsymbol{p}_{0}, t=0\right)=$ $F\left(\boldsymbol{x}_{0}, \boldsymbol{p}_{0}\right)$. Note that $\mathrm{d} \boldsymbol{x} \mathrm{d} \boldsymbol{p}=\mathrm{d} \boldsymbol{x}_{0} \mathrm{~d} \boldsymbol{p}_{0}$, i.e., the Jacobian of the coordinate transformation in phase space from $\boldsymbol{x}, \boldsymbol{p}$ to $\boldsymbol{x}_{0}, \boldsymbol{p}_{0}$ is equal to unity (corresponding to the invariance properties of one of the so-called Poincaré integrals). Clearly in the $\boldsymbol{x}_{0}, \boldsymbol{p}_{0}$ coordinates the Vlasov equation reads

$\frac{\partial F}{\partial t}=0$

where the partial time derivative is taken at constant $\boldsymbol{x}_{0}, \boldsymbol{p}_{0}$.

Suppose we are interested in studying the non-conservation of a given quantity $Q$ (which might be the single-star energy or radial action). Then we may introduce a different coordinate system in phase space where instead of $\boldsymbol{x}_{0}, \boldsymbol{p}_{0}$ we introduce the pair $Q_{0}, Q$, where $Q=Q(t) \equiv Q(\boldsymbol{x}(t), \boldsymbol{p}(t), t)$ is the appropriate function of the phase space coordinates $\boldsymbol{x}, \boldsymbol{p}$ and of time and $Q_{0}=Q(t=0)$, supplemented by four initial coordinates, which we symbolically denote by $\alpha_{j}$, with $j=1, \ldots, 4$. If $Q$ is not conserved, we expect that for $t \neq 0$ the Jacobian $\mathcal{J}=\mathcal{J}\left(Q_{0}, Q, \alpha_{j} ; \boldsymbol{x}_{0}, \boldsymbol{p}_{0}\right)$ be different from zero; but at $t=0$ we have $Q=Q_{0}$, so that the coordinate transformation becomes singular and $\mathcal{J}=0$. Clearly, if $Q$ were a conserved quantity the above coordinate transformation would be singular at all times; a similar situation may occur in the presence of an adiabatic invariant, when a quantity $Q$, such as the single-particle energy, is uniquely determined once $Q_{0}, \alpha$, and $t$ are given. In addition, the Jacobian $\mathcal{J}$ may vanish at special values of $t$, for special values of $Q_{0}, Q$ and $\alpha_{j}$, when a "caustic" type behaviour occurs.

In terms of the coordinates $Q_{0}, Q, \alpha_{j}$ the Vlasov equation for $\hat{F}=\hat{F}\left(Q_{0}, Q, \alpha_{j}, t\right)=F\left(\boldsymbol{x}_{0}, \boldsymbol{p}_{0}\right)$ reads

$\frac{\partial \hat{F}}{\partial t}+\frac{D Q(t)}{D t} \frac{\partial \hat{F}}{\partial Q}=0$

where $D Q(t) / D t$ is to be expressed as a function of $Q_{0}, Q, \alpha_{j}$, and $t$. Here the partial time-derivative is taken at constant 
$Q_{0}, Q, \alpha_{j}$. In terms of $\hat{F}$, the mass density $f(\boldsymbol{x}, \boldsymbol{p}, t) \mathrm{d} \boldsymbol{x} \mathrm{d} \boldsymbol{p}$ becomes

$f(\boldsymbol{x}, \boldsymbol{p}, t) \mathrm{d} \boldsymbol{x} \mathrm{d} \boldsymbol{p}=\frac{\hat{F}\left(Q_{0}, Q, \alpha_{j}, t\right)}{\mathcal{J}\left(Q_{0}, Q, \alpha_{j}\right)} \mathrm{d} Q_{0} \mathrm{~d} Q \mathrm{~d} \alpha_{j}$.

The general solution of Eq. (A.4) can be written as

$\hat{F}\left(Q_{0}, Q, \alpha_{j}, t\right)=\mathcal{F}\left(Q_{0}, \alpha_{j}, K_{\alpha_{j}}\left(Q_{0}, Q, t\right)\right)$,

where the characteristic $K_{\alpha_{j}}\left(Q_{0}, Q, t\right)$ is a solution of Eq. (A.4) at fixed $\alpha_{j}$.

An explicit solution of the above equations is given at the end of this Appendix for the special case of a one-dimensional motion in a time dependent but spatially uniform force. Here we consider two paradigmatic examples. Let us first consider the case of

$$
\frac{D Q(t)}{D t}=\frac{\left(Q-Q_{0}\right)}{t},
$$

which is expected to hold as typical behavior in the initial stages of the evolution, i.e., for $t \rightarrow 0$. At fixed $Q_{0}$, the characteristic is then given by

$K_{\alpha_{j}}\left(Q_{0}, Q, t\right)=\frac{\left(Q-Q_{0}\right)}{t}$,

so that the general solution for $\hat{F}$ should be written as a function $\mathcal{F}$ of the variables $Q_{0}, \alpha_{j}$, and $K_{\alpha_{j}}$ :

$\hat{F}\left(Q_{0}, Q, \alpha_{j}, t\right)=\mathcal{F}\left(Q_{0}, \alpha_{j},\left(Q-Q_{0}\right) / t\right)$.

Equation (A.9) provides the generic form of the distribution function in evolution variables for early times such that $(Q-$ $\left.Q_{0}\right) / Q_{0} \ll 1$ and shows that if the initial distribution function is only a function of $Q_{0}$ and of $\alpha_{j}$, it remains independent of $Q$ at all times (over the allowed domain of $Q$ at time $t$ ).

In the second example we take

$\frac{D Q(t)}{D t}=Q \frac{D \ln \left(\omega\left(Q_{0}, \alpha_{j}, t\right)\right)}{D t}$,

we have

$K_{\alpha_{j}}\left(Q_{0}, Q, t\right)=Q / \omega$

where $\omega=\omega\left(Q_{0}, \alpha_{j}, t\right)$ is an effective "frequency" and the allowed range of $Q$ at fixed $Q_{0}, \alpha_{j}$ is restricted to the single value $Q=\omega Q_{0} / \omega_{0}$, with $\omega_{0}=\omega\left(Q_{0}, \alpha_{j}, t=0\right)$. Therefore, in this case we find $Q-Q_{0}=Q_{0}\left(\omega / \omega_{0}-1\right)$, which compared with Eq. (A.9), illustrates the fact that in the presence of an adiabatic invariant the transformation from $\boldsymbol{x}, \boldsymbol{p}, t$ to $Q_{0}, Q, \alpha_{j}, t$ is singular at all times. In turn, finding a high concentration of points at all times along a curve in a scatter plot would suggest that the transformation be close to singular, which provides us with a global test of adiabatic invariance.

The scatter plots mentioned in Sect. 2.4 and used in Sect. 4 thus correspond to the mass density $D\left(Q_{0}, Q, t\right)$ in the $\left(Q_{0}, Q\right)$ plane obtained by integrating at time $t$ the mass density in phase space over the coordinates $\alpha_{j}$

$D\left(Q_{0}, Q, t\right)=\int \mathrm{d} \alpha_{j} \frac{\mathcal{F}\left(Q_{0}, \alpha_{j}, K_{\alpha_{j}}\left(Q_{0}, Q, t\right)\right)}{\mathcal{J}\left(Q_{0}, Q, \alpha_{j}\right)}$.

For $t \rightarrow 0$ we have

$D\left(Q_{0}, Q, t\right)=\frac{\mathcal{D}\left(Q_{0},\left(Q-Q_{0}\right) / t\right)}{t}$.
The framework that we have developed suggests a number of tests and of scatter plots based on different variables, especially in the limit $t \rightarrow 0$. We postpone these investigations to a separate paper, because they touch on issues that are not of immediate astrophysical interest, beyond the main goals of this article.

To conclude, we exemplify the theoretical framework just outlined by considering one simple time-dependent system for which the characteristics can be easily computed.

We consider the one-dimensional motion of a star in a homogeneous time dependent gravitational field described by the Hamiltonian

$H(x, p, t)=p^{2} / 2-2 x t$.

The characteristics are easily computed and are given by

$p=p_{0}+t^{2}$

$x=x_{0}+p_{0} t+t^{3} / 3$,

so that

$H\left(x_{0}, p_{0}, t\right)=p_{0}^{2} / 2-2 x_{0} t-p_{0} t^{2}-t^{4} / 6$.

Note that $H_{0}=H_{0}\left(x_{0}, p_{0}, t=0\right)=p_{0}^{2} / 2$.

We now focus on the non-conserved quantity $H$ (which was called $Q$ above) and consider the transformation $\left(x_{0}, p_{0}\right) \rightarrow$ $\left(H_{0}, H\right)$, which is easily recovered from:

$p_{0}=\left(2 H_{0}\right)^{1 / 2}$,

$x_{0}=\left(H_{0}-H\right) /(2 t)-\left(2 H_{0}\right)^{1 / 2} t / 2-t^{3} / 12$.

Here the square roots are properly defined so as to keep track of the sign of $p_{0}$. From Eq. (A.16) we obtain

$\frac{D H}{D t}=-2 x_{0}-2 p_{0} t-\frac{2 t^{3}}{3}=-2 x=\frac{\partial H}{\partial t}$,

which can be rewritten as

$\frac{D H}{D t}=\frac{H-H_{0}}{t}-\left(2 H_{0}\right)^{1 / 2} t-\frac{t^{3}}{2}$,

and reduces to $D H / D t \sim\left(H-H_{0}\right) / t$ for $t \rightarrow 0$. The characteristic function $K\left(H_{0}, H, t\right)$ defined by Eq. (A.4) is given by

$$
\begin{aligned}
K\left(H_{0}, H, t\right) & =\left(H_{0}-H\right) /(2 t)-\left(2 H_{0}\right)^{1 / 2} t / 2-t^{3} / 12 \\
& =x_{0}\left(H_{0}, H, t\right)
\end{aligned}
$$

as can be easily verified by substitution and is a priori obvious from the fact that, at constant $H_{0}$, the characteristics are labelled by the value of $x_{0}$.

The Jacobian $\mathcal{J}\left(H_{0}, H\right)$ is given by

$\mathcal{J}\left(H_{0}, H\right)=2\left|p_{0} t\right|=2\left(2 H_{0} t^{2}\right)^{1 / 2}$.

Thus from the distribution function $F\left(x_{0}, p_{0}\right)$, in the $H, H_{0}, t$ variables we have

$F\left(x_{0}, p_{0}\right) \mathrm{d} x_{0} \mathrm{~d} p_{0}=\frac{\mathcal{F}\left(K\left(H_{0}, H, t\right),\left(2 H_{0}\right)^{1 / 2}\right)}{2\left(2 H_{0} t^{2}\right)^{1 / 2}} \mathrm{~d} H_{0} \mathrm{~d} H$,

with $K\left(H_{0}, H, t\right)$ defined by Eq. (A.20). Note that, if at $t=0$ the distribution function is homogeneous in space it is constant in $H$ at all times. This is best realized by considering a distribution that is Gaussian both in $p_{0}$ and in $x_{0}$, for which we obtain in $H, H_{0}, t$

$$
\begin{aligned}
& \exp \left(-p_{0}^{2} / 2-x_{0}^{2} / L^{2}\right) \frac{\mathrm{d} x_{0} \mathrm{~d} p_{0}}{L}= \\
& \quad \exp \left\{-\left[\left(H-H_{0}\right) /(2 t)+\left(2 H_{0}\right)^{1 / 2} t / 2+t^{3} / 12\right]^{2} / L^{2}\right\} \\
& \quad \times \exp \left(-H_{0}\right) \frac{\mathrm{d} H_{0} \mathrm{~d} H}{2 L\left(2 H_{0} t^{2}\right)^{1 / 2}},
\end{aligned}
$$


which becomes independent of $H$ in the limit $L \rightarrow \infty$. For finite $L$, in the limit $t \rightarrow \infty$ the distribution function is centered around $H=-t^{4} / 6$ and has a width that increases much slower, as $t^{1 / 2}$, i.e., the distribution tends to become "monochromatic".

Two different examples, the case of a parametric linear oscillator and the case of a free star in a small-amplitude wavelike potential, have also been solved explicitly in this framework, demonstrating how adiabatic invariance, the formation of caustics, and the effects of resonances may be recognized by means of studies of the Vlasov equation in evolution variables.

\section{References}

Bertin, G., Liseikina, T., \& Pegoraro, F. 2003, A\&A, 405, 73 (Paper I) Blumenthal, G. R., Faber, S. M., Flores, R., \& Primack, J. R. 1986, ApJ, 301, 27 Bontekoe, Tj. R. 1988, Ph.D. Thesis, Groningen University, Groningen

Bontekoe, Tj. R., \& van Albada, T. S. 1987, MNRAS, 224, 349

Cipollina, M., \& Bertin, G. 1994, A\&A, 288, 43

El-Zant, A., Shlosman, I., \& Hoffman, Y. 2001, ApJ, 560, 636

El-Zant, A., Hoffman, Y., Primack, J., Combes, F., \& Shlosman, I. 2004, ApJ, 607, L75
Ghigna, S., Moore, B., Governato, F., et al. 2000, ApJ, 544, 616

Gnedin, O. Y., Kravtsov, A. V., Klypin, A. A., \& Nagai, D. 2004, ApJ, 616, 16 Goldstein, H. 1980, Classical Mechanics (Addison-Wesley, Reading), 2nd ed.

Goodman, J., \& Binney, J. 1984, MNRAS, 207, 511

Kochanek, C. S., \& White, M. 2001, ApJ, 559, 531

Landau, L., \& Lifchitz, E. 1969, Mécanique (Moscou: Éditions MIR)

Ma, C.-P., \& Boylan-Kolchin, M. 2004, Phys. Rev. Lett., 93, 021301

Merritt, D., \& Quinlan, G. D. 1998, ApJ, 498, 625

Mo, H. J., Mao, S., \& White, S. D. M. 1998, MNRAS, 295, 319

Moore, B., Governato, F., Quinn, T., Stadel, J., \& Lake, G. 1998, ApJ, 499, L5

Navarro, J. F., Frenk, C. S., \& White, S. D. M. 1996, ApJ, 462, 563

Navarro, J. F., Hayashi, E., Power, C., et al. 2004, MNRAS, 349, 1039

Nipoti, C., Treu, T., Ciotti, L., \& Stiavelli, M. 2004, MNRAS, 355, 1119

Northrop, T. G. 1963, The Adiabatic Motion of Charged Particles (New York: Wiley Interscience)

Quinlan, G. D., Hernquist, L., \& Sigurdsson, S. 1995, ApJ, 440, 554

Trenti, M. 2005, Ph.D. Thesis, Scuola Normale Superiore, Pisa

Trenti, M., \& Bertin, G. 2005, A\&A, 429, 161

Trenti, M., Bertin, G., \& van Albada, T. S. 2005, A\&A, 433, 57

van Albada, T. S. 1982, MNRAS, 201, 939

van der Marel, R. P. 1999, AJ, 117, 744

Young, P. 1980, ApJ, 242, 1232

Zaritsky, D., \& White, S. D. M. 1988, MNRAS, 235, 289 